\title{
TTR
}

Traduction, terminologie, re?daction

\section{Mallarmé visible et invisible}

\section{Serge Gavronsky}

Volume 12, numéro 1, 1er semestre 1999

Poésie, cognition, traduction I

Poetry, Cognition, Translation I

URI : https://id.erudit.org/iderudit/037355ar

DOI : https://doi.org/10.7202/037355ar

Aller au sommaire du numéro

\section{Éditeur(s)}

Association canadienne de traductologie

ISSN

0835-8443 (imprimé)

1708-2188 (numérique)

Découvrir la revue

Citer cet article

Gavronsky, S. (1999). Mallarmé visible et invisible. TTR, 12(1), 115-130.

https://doi.org/10.7202/037355ar

\section{Résumé de l'article}

Mallarmé visible et invisible - Le grand poète américain Louis Zukofsky, fondateur de l'école dite Objectiviste, dans la lignée de l'épique, incorpore la totalité du vécu. Dans cette vaste entreprise, intitulée « A ", Mallarmé figure brièvement mais jamais jusqu'à présent n'a-t-on relevé les nombreuses naturalisations textuelles où le poète français, « translaté » en anglais dans "A »-19 (sans attribution aucune), non seulement figure dans l'oeuvre zukofskienne mais l'inspire profondément.
Tous droits réservés (C) TTR: traduction, terminologie, rédaction — Les auteurs, 1999
Ce document est protégé par la loi sur le droit d'auteur. L’utilisation des services d'Érudit (y compris la reproduction) est assujettie à sa politique d'utilisation que vous pouvez consulter en ligne.

https://apropos.erudit.org/fr/usagers/politique-dutilisation/ 


\section{Mallarmé visible et invisible}

\section{Serge Gavronsky}

N'y aurait-il pas un certain paradoxe dans le postulat d'une lecture de l'invisible? L'évidence ne soutient guère cette supposition car, depuis l'invention de l'écriture, des initiés se prêtent à ce type de décodage : par exemple, le déchiffrement de la Bible par les rabbins. De nos jours une lecture psychanalytique ou marxiste ne voit aucun obstacle dans l'épuisement d'un texte qui serait autrement « innocent ». D'ailleurs dans le domaine de la poésie, l'interprétation occupe un espace privilégié : qu'il s'agisse d'appliquer des théories qui s'appuient sur la linguistique, la sémiotique, ou la rhétorique, aucune n'hésite devant ce qu'on aurait pu considérer comme de l'invisible. À chacun donc d'aborder ce territoire pour en faire valoir un sous-texte ou en tout cas éclaircir ce qui se trouve caché à la surface du texte.

Un simple regard ( $y$ en a-t-il encore?) jeté sur la peinture surréaliste, sur les toiles des métaphysiques italiens, sur les collages d'un Braque ou d'un Picasso met en jeu le désir d'aller au-delà de l'évidence. C'est alors que nous nous posons certaines questions : quel est le sens de cette inscription apparemment hermétique? Existe-t-il un dédoublement du sens? La forme est-elle porteuse de sens? Et peu à peu nous apprenons comment nous y prendre afin de satisfaire une curiosité bien nourrie par les pratiques de notre siècle.

Ainsi une poétique/poésie qui de prime abord semble être indéchiffrable, celle de Louis Zukofsky, poète appelé par Hugh Kenner le « Mallarmé américain "1, nous interpelle, nous arrête également, car le

I Voir la quatrième page de couverture de Louis Zukofsky, Bottom : On Shakespeare, Berkeley, University of California Press, 1963. 
mixage de codes linguistiques, les allusions musicales, mathématiques, scientifiques et politiques, mais aussi les interférences autobiographiques et littéraires rendent son poème épique $\| A$ » de prime abord... illisible, voire invisible dans le sens profond qui le détermine. Par la suite, entrés dans la matière chronologique (le poème, commencé en 1928, est achevé en 1974), nous sommes suffisamment curieux pour interroger l'Index de l'édition définitive?. Une foule de noms propres se succèdent parmi lesquels celui de Stéphane Mallarmé.

Jusqu'à présent le seul à avoir systématiquement analysé ce poème n'a trouvé autre chose à dire que ceci : Louis Zukofsky « jouait sur Mallarmé comme son fils, Paul, sur un violon qui appartenait à Paganini $»^{3}$. Vingt-cinq pages plus loin (1983, p.166), cette autre appréciation : Mallarmé et Zukofsky furent, tous les deux, professeurs d'anglais. Un point c'est tout. Et ce « tout " se trouve dans la séquence "A 》-19 (le poème en contient 24). Rien n'est donc moins difficile pour un lecteur en quête d'un Mallarmé et aucune déception possible : nous le trouvons quatre fois nommé dans le texte et par la suite, métonymiquement représenté par $E$ et Livre, deux termes écrits en français dans le poème. À la surface du texte plane donc le poète français, sa poétique, sa pensée : sa présence est irrécusable. Nous sommes heureux : le travail a été fait par un critique avisé - Mallarmé figure bel et bien dans l'œuvre du poète américain, né dans le Lower East Side en 1904 et mort dans sa ville natale en 1978. Ce même poète - pour le présenter - fut le fondateur de l'école Objectiviste, et l'ami d'Ezra Pound, de William Carlos Williams, de Basil Bunting et plus tard d'Allen Ginsberg et de Robert Creeley. Mais une simple audition du texte laisse rêver un lecteur français, car les traces mallarméennes traversent la séquence; oui, insoupçonnées, elles ne portent aucun signe d'identité sauf de simples guillemets, suffisamment présents, néanmoins, pour nous convaincre que Stéphane Mallarmé s'inscrit majestueusement dans l'œuvre de Louis Zukofsky. Et ce sera l'objet de ma lecture.

Ne faudrait-il pas remonter aux études poursuivies à Columbia College, Columbia University, pour vérifier si déjà, jeune universitaire,

2 Louis Zukofsky, A, Berkeley, University of California Press, 1978. Toutes les citations sont tirées de cette édition.

3 Barry Ahearn, An Introduction. Louis Zukofsky's A, Berkeley, University of California Press, 1983, p. 144. 
Zukofsky n'aurait pas rencontré le poète français? Mon hypothèse est qu'il aurait pu lire le texte initiatique en la matière, le livre d'Arthur Symmons, The Symbolist Movement in Literature (1899) qui a tant marqué T. S. Eliot. Ce n'est là évidemment qu'une simple hypothèse. Par contre, on retrouve Mallarmé cité dans l'article " The Writing of Guillaume Apollinaire " publié dans The Westminster Review (spring 1933/winter 1934) où Zukofsky nous rappelle que l'absence de ponctuation remonte à Mallarmé. Par la suite, on le rencontre à nouveau, mais cette fois-ci en bloc, dans Bottom : On Shakespeare. Zukofsky y compose une minianthologie de la poésie française partant de Malherbe pour aboutir à Jean Genet. Mallarmé y figure avec cinq poèmes cités sous forme de fragments et en français : Pitre châtié, Don du poème, L'Après-midi d'un Faune, Prose, et Feuillet d'album. Ces textes figurent tous dans un recueil sur Mallarmé que possédait Louis Zukofsky : les traductions de Roger Fry4. À l'exception de Feuillet d'album, tous ces poèmes sont accompagnés de commentaires détaillés. Ce même ouvrage de Roger Fry sera donné par Zukofsky à son fils avec sur la deuxième page de couverture la mention : "Louis Zukofsky, May 17, 1966, for Paul. »

D'autres traductions figurent sur les étagères de Zukofsky, notamment deux travaux importants : C. F. Mclntyre, The Selected Poems of Stéphane Mallarmés avec une préface utile et Un coup de dés jamais $n^{\prime}$ abolira le hasard traduit par Daisy Aldan'. Selon le critique Kenneth Cox, Zukofsky aurait été également un fin lecteur du Guignon et du Placet futile de Mallarmé $e^{7}$. Si Cox situe ces deux poèmes dans le texte de Zukofsky ainsi que des emprunts puisés dans l'étude de Jacques Schérer, par contre, comme je vais le signaler plus loin, il n'a aucunement relevé d'autres indices d'une importance majeure.

4 Roger Fry, Mallarmé, New York, New Directions, 1951.

${ }^{5}$ C.F. McIntyre, The Selected Poems of Stéphane Mallarmé, Berkeley, University of California Press, 1957.

6 Daisy Aldan, trans. Un coup de dés jamais n'abolira le hasard, New York, Folder Editions, 1961.

7 Kenneth Cox, «Zukofsky and Mallarmé : Notes on " $A$ " -19, " in Maps \#5 Louis Zukofsky, Athens, Ohio, The Lawhead Press, 1973, pp. 1-12. 
Un autre élément entre en jeu. Paul Zukofsky, le fils, enfant prodige et violoniste renommé, revient de Paris où il a participé au concours Jacques Thibaud (17/11/63) en rapportant à son père l'étude de Jacques Schérer, Le "Livre " de Mallarmés. Le père, nous l'avons vu, reconnaîtra sa dette envers son fils, car c'est bien grâce à Paul Zukofsky que Louis prendra connaissance de cette analyse fondamentale. Pourquoi le fils aurait-il été intéressé par le poète français? Depuis plusieurs années, Paul Zukofsky est fasciné par l'art de la composition dans ses états les plus modernes. C'est ainsi qu'il tient compte de Pierre Boulez qui venait d'écrire deux improvisations sur les poèmes de Mallarmé : Le Vierge, le vivace et le bel aujourd'hui et Une dentelle s'abolit. Une visite chez le libraire Brentano, avenue de l'Opéra, lui permet d'acheter l'étude de Schérer qu'il allait, par la suite, offrir à son père. Dans la séquence du poème que nous allons étudier, c'est-à-dire " $A \|-19$, le père dit très peu de choses sur Mallarmé, mais lors d'un entretien le fils soulignera avec véhémence : " Papa empruntait sans jamais identifier ces écrits. »"

Regardons maintenant le poème « $A$ »-19, commencé en 1965 et terminé l'année suivante. Quiconque se mettrait un tant soit peu à l'écoute de Mallarmé, prosateur et poète, ne saurait éviter cette rencontre avec son œuvre ici recomposée, repensée, je dirais traduite comme le voulaient saint Jérôme, Cicéron et Horace, c'est-à-dire non seulement neutralisée mais naturalisée. Ainsi, grâce aux bons soins de Louis Zukofsky, Stéphane Mallarmé est assuré d'une citoyenneté américaine : il passera, inaperçu, dans cet univers d'outre-mer.

Faudrait-il commencer avant même le commencement? Faire un rapprochement peut-être inattendu sous la forme d'un chiasme c'est-àdire, d'un double chassé-croisé entre le père et le fils ? Zukofsky écrit :

Son with

concert shoes

that you

bring me

Le Livre

« $A »-19,421$

8 Jacques Schérer, Le « Livre » de Mallarmé, Paris, Gallimard, 1957.

9 Entretien avec Paul Zukofsky, 26 mai 1998. Je tiens ici à remercier Paul Zukofsky pour tout ce qui relève des renseignements bio-bibliographiques. 
Là où Mallarmé avait écrit :

Faisant revivre

.......construire

avec sa (netteté)

lucidité - cette

œuvre - trop

vaste pour moi.10

Ou encore :

moi, sans toi

(Richard 161)

Une hantise : le père accorde à son fils ses propres attributs - sans vouloir suggérer qu'un Zukofsky s'accusait du sort de son fils Paul! Un point de départ en tout cas. En voici un autre : Mallarmé écrit dans "Quant au livre " : "Le livre, expansion totale de la lettre... " " Y auraitil une parole plus apte pour définir le projet de Zukofsky qui inaugure son œuvre en parfaite résonance avec cette déclaration :

A

round of fiddles

playing Bach

« $A \rrbracket-1$

Donc... de " $\mathrm{A}$ » à « $\mathrm{B}$ » pour Bach! Et encore, quand Mallarmé écrit : "Enfin du moi - et du langage mathématique. " $(O C, 851)$ J'insisterais ici sur une passion semblable chez Zukofsky qui, tout au long de « $A$ », souligne l'importance du chiffre (rappel de la Kabbale et de Baudelaire), notamment dans les formules suivantes :

(bless us)

divine number begins

« $A »-19,419$

Et avec plus de précision :

10 Jean-Pierre Richard, Stéphane Mallarmé et son fils. Paris, Éditions du Seuil, 1961, p. 112.

"I Stéphane Mallarmé, CEuvres complètes, texte établi et annoté par Henri Mondor et G. Jean-Aubry , Paris, Gallimard, coll. « La Pléiade », 1945, p. 380. 
you should

have patience

ranging random

numbers...

pour en conclure :

..singing harmonies

of seven vowels

« $A \rrbracket-19,420$

Le lien entre parole, chiffre et musique se lit à travers le poème de Zukofsky, qu'il s'agisse de numérologie, d'une appréciation portant sur la lumière, ou encore de cette interrogation zukofskienne qui serait une théorie effleurée d'une traduction intersémiotique :

Can

The design

Of the fugue

Be transferred

To poetry?

$$
\text { « } A »-6,38
$$

L'évidence : en premier lieu, Zukofsky valorise les éléments formels dans la composition de son " Livre » en 24 séquences. Il interroge le métier du poète, soumet son propre travail à un regard ironique quand il mime la voix de son fils ( $A »-12$ ). Bien entendu, l'écrit dépend également d'une inscription qui incorpore l'histoire de son siècle, et une idéologie anti-poundienne où le Mus de l'un devient le Lénine de l'autre. Malgré ces divergences d'avec la poétique mallarméenne, l'identité de l'œuvre de Zukofsky est semblable à celle de Mallarmé. Jacques Schérer écrit : « Le Livre est théâtre, il est poésie, il est prose, il est même journal, rien de ce qui est littéraire ne peut lui être étranger " (Schérer, Il). Zukofsky n'ajoute qu'un référent fondamental quand il inscrit dans la poésie ce qu'elle n'avait antérieurement toléré : le vulgaire, l'oralité, le mixage de registres linguistiques partant de l'argot, des idiolectes jusqu'au sublime sous forme de sonnets shakespeariens dans " $A$ » -7 et " $A \|-9$. Imiter le modèle puis le dépasser. Cependant, on retrouve chez Zukofsky cette version du texte de Schérer (Kenneth Cox l'a constaté) :

...Piece or

that play

with concert 
dialog poem

..symphony for

scene.

$$
\text { « } A »-19,423
$$

Et quoi de plus frappant dans cette vision globale qu'Un coup de dés jamais n'abolira le hasard? Ce poème, épique lui aussi, se retrouve en filigrane dans « $A$ » où " flipping a coin » (《 $A$ »-19,429) remplace le coup de dés, substitution idéologique dans la tradition whitmanienne : l'un, noble, se voit déplacé par une pièce de monnaie roturière. Ajoutons à ce rappel, non seulement l'évocation du « hasard », écrit en français dans le texte ( $A$ $A$ » 19, 422) mais par la suite, " his century's dice » (" $A »-19$, 422). Ce qui est certain : le geste mallarméen est traduit par le terme «forecasting » qui, lui, se prête également à une autre tournure permettant le " casting foreward ".

Mais il s'agit de la totalité de l'entreprise mallarméenne, et Zukofsky écrit :

But how
beautiful a
last mind
dies : 'What book?
what book?
entire enough
perfect enough
to take
the place
of all
the books
and of
the world itself

« $A$ $)-19,423$

Où, sinon dans les écrits de Mallarmé et de Schérer, Zukofsky aurait-il pu découvrir cette définition totalisante qui correspond si merveilleusement à celle de Mallarmé et de sa conception du Livre comme étant la textualisation ainsi qu'une incorporation absolue du monde? 
Et Zukofsky ajoute :

Mallarmé's late

thoughts minds

'the book

however seeming

never begins

or ends

...the crowd

other than

by silence

takes part

exults as

choir..voice..vaults'

« $A \rrbracket-19,426$

Ai-je entendu un écho de Hamlet dans ce texte? Se peut-il, comme le suggère Philippe Sollers, que Shakespeare avec Descartes aient été les deux inspirations principales de Mallarmé? ${ }^{12}$ Que Shakespeare ait été l'un des fondements de la poétique zukofskienne est indubitable. Très jeune, accompagné par son frère aîné, Louis Zukofsky fréquentait le théâtre Yiddish de la Seconde Avenue dans le Lower East Side : il a vu, en traduction, les plus grandes pièces du maître anglais. Autre rappel biographique : à la suite d'une suggestion faite par son institutrice, à l'âge de onze ans, Louis avait déjà lu le théâtre de Shakespeare dans son intégralité. Par ailleurs, Mallarmé écrit dans Les Mots anglais où il établit une double tradition, anglaise et américaine : " Shakespeare, Milton, Shelley et Byron et tant de merveilleux prosateurs, voilà des génies qui se sont, à travers les siècles, transmis le trésor double du langage ici étudié " $(O C, 914)$. Et Mallarmé ajoute à cette liste, Poe et Longfellow $(O C, 890)$. Comment ne pas retrouver dans ce " silence " chez Zukofsky la fin de Hamlet et dans cette exaltation du chœur, des voix et des voûtes, les éléments gothiques d'un dix-neuvième siècle poétisé?

Les affinités sont nombreuses. Zukofsky insiste sur une réelle filiation quand il écrit :

Stéphane Mallarmé

12 Philippe Sollers, L'écriture et l'expérience des limites. Paris, Éditions du Seuil, coll. «Tel Quel », 1968, p. 70. 
whose book

prophecy say

his branch

bring to

our family

« $A$ » $-19,427$

En fait, deux Livres et une connexion qui suggère l'ancienneté de Mallarmé, prophète dans son droit, Père en majuscule, c'est-à-dire, initiateur : l'origine tant recherchée depuis la Genèse. Mais la descendance généalogique est américanisée : du haut de ce Panthéon la bonne nouvelle atteint la « famille ». Celle des poètes? Sa propre famille d'Hester Street? Et pourquoi choisir entre les deux?

\section{Deux traducteurs}

Nous le savons tous : pour de nombreuses raisons, Mallarmé a entrepris des traductions de l'anglais en français, et si Edgar Poe, à la suite de Baudelaire, retient notre admiration, il ne faudrait pas oublier d'autres traductions qui ont été entreprises pour s'assurer des revenus supplémentaires, comme cette longue prose qui met fin à l'édition de la Pléiade, L'étoile des Fées, d'un auteur qui n'existait qu'à peine : Mrs. W.C. Elphinstone Hope. L'autre écrit, Les Dieux antiques, trace l'origine et le développement de la mythologie, examine les mythes hindous, nordiques et persans, et représente à nouveau les mythes grecs et latins. Ce qui attire mon regard serait plutôt l'avant-propos de l'éditeur, car dans ses commentaires, il articule une théorie de la traduction qui remonterait à saint Jérôme. Cet anonyme écrit : « Impossible, même dans un travail de traduction, que la présence de l'esprit français ne se fasse remarquer... " $(O C, 1159)$. Voilà une lecture impérialiste! Lecture qui semble priser non seulement le contenu mais surtout la forme qui trahit ses origines, peutêtre même sa mythologie, c'est-à-dire, cet indéfinissable "esprit français " qui caractérise, pour ne pas aller au-delà du dix-neuvième siècle, l'une des raisons d'être de la " civilisation » française. Est-ce par opposition à la kulıur germanique? En tout cas, Mallarmé en est le maître accompli. À cet effort qui serait comme une " francisation ", Mallarmé dans ses Thèmes anglais aborde un autre discours, et celui-là coexistensif à celui de Louis Zukofsky, par l'importance accordée au signifiant comme porteur de sens. Un exemple suffira. En évoquant le sens des sons, du son $s$, Mallarmé écrit : " [...] le sens principal peut-être de la lettre $s: s t$ 
s'adjoint même $r$ (en str) pour dire force, élancement et par suite errer et joncher. » Il ajoutera que " [...] sc ainsi que $s n$, rejetés par le français notamment, ne se présentent en anglais que sous un mauvais jour : ici montrant les idées de faiblesse et de lâcheté $[\ldots]$ ". $(O C, 948)$.

Cette redéfinition du signifiant est partagée par Zukofsky et j'en offrirai deux exemples. Dans un premier temps, à la suite d'Ezra Pound, Zukofsky traduit la donna mi Prega de Guido Cavalcanti, mais prenant le "Make it New! " de Pound au sérieux, ce poème se retrouve surnaturalisé américain, c'est-à-dire, traduit avec les accents de Brooklyn, ce qui donne " A Foin Lass Bodders 》13. Dans un deuxième temps (serait-il à l'écoute mallarméenne?), avec sa femme, Celia, il se lance dans une translittération des poèmes de Catulle ${ }^{14}$, travail d'amour, sans doute, marqué par un procédé radical où le son des mots latins suggère un sens équivalent en anglais.

Mais serais-je le premier à retrouver Mallarmé dans « $A$ » -19 à partir d'une prose bien connue?

Tu remarquas, on n'écrit pas, lumineusement, sur champ obscur, l'alphabet des astres, seul, ainsi s'indique, ébauché ou interrompu; l'homme poursuit noir sur blanc. $(O C, 370)$

Quand Zukofsky se met à l'œuvre il faut tenir compte de ses pratiques de traduction et ne pas espérer retrouver le texte d'origine reproduit tel quel dans la langue cible. Zukofsky ira plus loin en " traduisant » la prose en poésie, et allant encore plus loin, il reprendra à son compte le texte de Mallarmé sous une forme en parfait accord avec le reste de la séquence, par un procédé que l'on pourrait rapprocher d'une pratique citationnelle, ce qui donne :
...'Man
does not
write with
light on
black crystal

${ }^{13}$ Louis Zukofsky, First Half of « $A$ » -9, New York, Privately printed, 1940, pp. 35-36.

14 Louis Zukofsky et Celia Zukofsky, Gaius Valerius Catullus, London, Cape Goliard, 1969. 
night..in

black ink's

audacity..married

to his night.'

" $A »-19,425$

Certes nous retrouvons notre Mallarmé, mais n'est-il pas " translaté ", digéré pour ainsi dire par le corps-texte, au point de " disparaître ", ou tout au moins passer pour aussi hermétique que l'original? Zukofsky n'indique l'étrangeté de ce passage qu'avec des guillemets simples; le nom de l'auteur en est exclu comme dans un collage cubiste. Ajoutons que Zukofsky repense cet écrit canonique en nous proposant une lecture/traduction en biais, créant aussi un calembour dans la conclusion de cet emprunt : " married to his night ». Dans l'ordre homophonique pratiqué plus haut, ce " mariage " se prêterait à un double décodage : « night » et « knight », surtout à la suite du terme « audacity ».

Et Zukofsky va au devant de cette première évocation :

Is there

man ink

and does

his 'white

paper support'

eyes the

fine day

he'll look

away from

black letters

to regret

sun (window)

« $A »-19,425$

Centrées dans la colonne zukofskienne, les traces de Brise marine et de Fenêtres; un parfait témoignage de la complicité du poète-lecteur américain avec les intertextes français.

\section{Le Tombeau de Stéphane Mallarmé}

Que le lecteur se penche avec moi sur ce corps placé là par l'intermédiaire d'un autre, tout aussi illustre. Et je rappelle ici un texte de Freud : « By 
absorbing part of the body of a person through the act of eating we also come to possess the properties which belonged to that person $/ 15$. J'aurais pu également citer ces vers d'Ariel dans la Tempête de Shakespeare :

Full fathom five thy father lies;

Of his bones are coral made;

Those are pearls that were his eyes :

Nothing of him that doth fade,

But does suffer a sea-change

Into something rich and strange.

(I, ii, 396-401)

Le choix nous appartient : Freud ou Shakespeare. En fait, Freud lui-même citait ce même passage de Shakespeare et donc nous le retrouvons chez Mallarmé et chez Zukofsky comme chez notre psychanalyste. Incontestables sont les « restes ", les " fragments » de l'original, peu importe le procédé de translation. Mallarmé nous propose un tel exercice, semblable à une explication de texte, quand il traduit en anglais $L e$ tombeau d'Edgar Poe et y ajoute une glose expliquant ce qu'il croyait incompréhensible en Amérique. Il s'adresse à Sarah Helen Whitman, femme à laquelle Poe aurait proposé le mariage. Je cite d'abord l'explication de Mallarmé : « naked hymn means when the words take in death their absolute value 》 et par la suite sa propre traduction des deux vers : " But, in a vile writhing of an hydra (they) once hearing the angel/To give too pure a meaning to the words of the tribe... " Tentation pédagogique : en 1940, selon une motivation pas trop éloignée de celle d'un Mallarmé qui voulait " éclaircir » le sens de son sonnet, Zukofsky publie, à titre d'auteur, une explication de texte : The First Half of " $A$ »-9. Dans une section intitulée "Restatement ", Zukofsky exprime une certaine angoisse devant l'impossibilité de se faire comprendre par le commun des mortels. Il écrit : " the poem sings about things embodying a common denominator of past work, tho this abstract evaluation of them hides the fact that things are goods made to be used by people " (Zukofsky, First half of " $A »-9,40$ ). Qui resterait indifférent à cet alliage pongien de Mallarmé et de Marx où la mention explicite de l'invisible, voire de ce qui est " caché ", appelle une clarification sur un mode qui double la traduction et la glose mallarméennes? Ajoutons : Zukofsky

15 Sigmund Freud, Totem and Taboo, New York, Vintage Books, 1946, p. 107. 
insiste sur le référent marxisant, ce qui encadrerait une approche herméneutique.

Rappelons ici les deux vers dans leur version originale en français : « Eux, comme un vil sursaut d'hydre oyant jadis l'ange/Donner un sens plus pur aux mots de la tribu ", afin de mieux les " reconnaître » dans la traduction fidèle/infidèle de Zukofsky qui va les translater de la manière suivante :

professing to

make pure

the speech

of a

scrawling race

$$
\text { « } A \text { » }-19,421
$$

Ainsi Zukofsky cannibalise Mallarmé! Ainsi Mallarmé devient-il le festin secret d'un poète d'outre-mer! Ce qui attire l'attention? En premier lieu le verbe " professing " qui, suivant l'habitude de Zukofsky, relève simultanément de la "réalité » et de la rhétorique, ici parfaitement conjointes. Le glissement paradigmatique du verbe français « donner " vers son équivalent anglais sonne le glas de la mort du père, pour reprendre la formule d'Ariel. En deuxième lieu, si Mallarmé dans l'original (et non dans sa traduction fautive) hyperbolisait l'ambition d'aller plus loin dans cet effort de purification, l'anglais se satisfait d'une simple opération. Par opposition au " plus pur », condition caractéristique dans la lecture mallarméenne où il faut se dépasser, en anglais, il suffit de purifier, « make pure ".

J'aborderai maintenant la distinction la plus radicale dans la version (invisible, disons-le) de Zukofsky qui, au moyen d'un adjectif et d'un nom, modifie l'intention et la direction du texte initial. Que peut bien signifier "scrawling »? Je l'entends dans un sens derridien, celui du magma inaugural d'où sortiraient les premiers mots gribouillés, le tohubohu biblique, l'écriture au stade de son enfance : le signe inaugural même. Et si nous sommes attentifs à la sonorité, à un dédoublement du sens partant de ce singulier signifiant, cela donnerait " crawling ", renforçant ainsi l'hypothèse ci-dessus. N'oublions pas l'attention que Mallarmé portait à cette union de consonnes : sc! 
Que dire de cette substitution de « race " pour « tribu »? De cet échange sémantique aux parfums ethnographiques? On pourrait indiquer tout simplement que pour Mallarmé " tribu " représentait une microstructure qui, métonymiquement, évoquait les poètes eux-mêmes; et que Mallarmé avait besoin d'une rime pour son «bu »! Zukofsky parvient à ce terme sous l'influence, je le crois bien, de l'anthropologie qui naît à Columbia où l'on venait de nommer Franz Boas comme premier directeur des études d'anthropologie et, à ses côtés, un autre illustre ethnologue, Bronislaw Malinowski, tous deux particulièrement soucieux d'approfondir les définitions de race, culture et langage.

C'est insuffisant comme explication car Juif, Zukofsky ne pouvait échapper à cette autre inflexion connotative qui symbolise les Juifs comme la race du Livre. Si Mallarmé ignorait la Kabbale, comme d'ailleurs les lettrés de cette fin de siècle, Zukofsky, lui, l'avait maîtrisée dès sa tendre jeunesse, et il était parfaitement à l'aise dans trois langues : l'anglais, l'hébreu et le yiddish.

Pour conclure, Zukofsky ne peut se situer dans le texte antérieur sans y apporter sa propre interprétation. Ce travail comporte un démembrement de l'original, une restructuration matérielle sur la page, une réinterprétation textuelle qui permet toutefois aux lecteurs ayant Mallarmé en tête de reconnaître le poète français dans ses nouveaux habits américains. J'irai plus loin : si Mallarmé ne figure, n'agit qu'en tant que figure dans " $A \rrbracket-19$, son spectre hante la totalité de l'œuvre dans un double sens : Zukofsky dans " $A$ » rivalise, comme le fait aussi tout poème épique, avec la Genèse; il se prend pour le nommable, le créateur par excellence. Ainsi, le Livre n'est aucunement une simple production, c'est une vaste entreprise réglée par une pensée prospective, avec ses séquences, ses mots et surtout ses lettres et ses chiffres que Mallarmé avait griffonnés dans les notes qui définissaient le Livre à venir.

$\grave{A}$ un certain moment, Paul se moque de son père et lui dit : "Wait till they find out/Where you took most of 'your' poetry " ( $A$ " $-12,214)$. Nous le savons à présent. 


\section{Références}

AHEARN, Barry (1983). An Introduction. Louis Zukofsky's A. Berkeley, University of California Press, $254 \mathrm{p}$.

ALDAN, Daisy, trad. (1961). Un coup de dés jamais n'abolira le hasard. New York, Folder Editions.

COX, Kenneth (1973). "Zukofsky and Mallarmé : Notes on « $A$ " -19 " dans Maps \#5 Louis Zukofsky. Athens, Ohio, The Lawhead Press, pp. 112.

FREUD, Sigmund (1946). Totem and Taboo. New York, Vintage Books, $207 \mathrm{p}$.

FRY, Roger (1951). Mallarmé. New York, New Directions, 312 p.

MALLARMÉ, Stéphane (1945). CEuvres complètes. Texte établi et annoté par Henri Mondor et G. Jean-Aubry. Paris, Pléiade/Gallimard, 1659 p.

McINTYRE, C. F. (1957). The Selected Poems of Stéphane Mallarmé. Berkeley, University of California Press, $169 \mathrm{p}$.

RICHARD, Jean-Pierre (1961). Stéphane Mallarmé et son fils. Paris, Éditions du Seuil, 315 p.

SCHÉRER, Jacques (1957). Le " Livre " de Mallarmé. Paris, NRF/Gallimard, $414 \mathrm{p}$.

SOLLERS, Philippe (1968). L'écriture et l'expérience des limites. Paris, coll. « Tel Quel » Éditions du Seuil, 190 p.

ZUKOFSKY, Louis (1978). A. Berkeley, University of California Press, $826 \mathrm{p}$.

- (1940). First Half of « $A »-9$. New York, Privately printed, 41 p.

- (1963). Bottom : On Shakespeare. Berkeley, University of California Press, 470 p. 
ZUKOFSKY, Louis et Celia ZUKOFSKY (1969). Gaius Valerius Catullus. London, Cape Goliard, 175 p.

RÉSUMÉ : Mallarmé visible et invisible - Le grand poète américain Louis Zukofsky, fondateur de l'école dite Objectiviste, dans la lignée de l'épique, incorpore la totalité du vécu. Dans cette vaste entreprise, intitulée " $A$ », Mallarmé figure brièvement mais jamais jusqu'à présent n'a-t-on relevé les nombreuses naturalisations textuelles où le poète français, " translaté » en anglais dans « $A$ »-19 (sans attribution aucune), non seulement figure dans l'œuvre zukofskienne mais l'inspire profondément.

ABSTRACT : Mallarmé Visible and Invisible - Major American poet and founder of the Objectivist School, Louis Zukofsky, adheres to the definition of the epic poem while incorporating the totality of his own history as well as of History itself. In " $A$ " Mallarmé figures both explicitly and metonymically, as well as in unsuspected ways. In fact, in Zukofsky's hands, Mallarmé is transfigured to the point of gaining a sort of American nationality, unsuspected until now, exceeding his acknowledged presence in " $A$ "-19.

Serge Gavronsky : Barnard College, Columbia University, 3009 Broadway, New York, NY 10027-6598.

Courriel : sgavronsky@Columbia.Barnard.edu 